\title{
Linking Consumer Opinions with Reservation Prices in an Agent-Based Model of Innovation Diffusion
}

\author{
A. Kowalska-Pyzalska ${ }^{a, *}, \mathrm{~K} . \mathrm{CWWIK}^{a}$, A. JęDrzeJewski ${ }^{a}$ And K. Sznajd-Weron ${ }^{b}$ \\ ${ }^{a}$ Department of Operations Research, Wrocław University of Technology, Wrocław, Poland \\ ${ }^{b}$ Department of Theoretical Physics, Wrocław University of Technology, Wrocław, Poland
}

\begin{abstract}
We extend a recently developed agent-based model of innovation diffusion by linking the opinions of potential consumers with their market behavior via the concept of reservation prices. Through a dynamic mechanism that takes into account social influence, the agents in our model can both increase or decrease their product appraisal. Considering complete graph network structures and using mean-field treatment we find that the model can exhibit a plethora of scenarios, observed empirically but not attainable within the classical Bass model. We also show the existence of a critical market price above which the innovation cannot diffuse.
\end{abstract}

DOI: 10.12693/APhysPolA.129.1055

PACS/topics: 89.65.Gh, 87.23.Ge

\section{Introduction}

The diffusion process of innovative products has been studied in the literature from various perspectives, including the impact of consumer heterogeneity, the role of social influence and the effectiveness of promotion and advertisement [1-3]. In this paper we extend a relatively simple, yet sufficiently rich agent-based model of opinion formation $[4,5]$ by linking the opinions of potential consumers with their market behavior via the concept of the reservation price, i.e. the highest price that an agent will accept and still purchase the good [6].

We focus on word-of-mouth (WOM) as the core social interaction, because it has been found to have a powerful influence on consumer behavior [2]. Empirical results indicate that there are two types of WOM: positive that enhances the diffusion by providing positive information about the product in the social network and negative that spreads the negative information about the product and limits diffusion $[7,8]$. The majority of the innovation diffusion literature focuses on positive WOM and on the benefits resulting from using a product. In reality, the innovation also has to face negative WOM, as well as the difficulty of adoption as perceived by consumers. This is especially important when the product is new so that its evaluation cannot be based on past experience.

To perform an in-depth study of the complex influence of WOM, similarly to $[5,7]$ and in contrast to most papers in the literature [9], we allow for opinion reversibility. Through a dynamic mechanism that takes into account social influence, the agents in our model can both increase and decrease their product appraisal. This opinion reversibility is supported by empirical studies. In particular, Rogers [1] argues that individuals seek reinforcement

\footnotetext{
* corresponding author; e-mail: anna.kowalska-pyzalska@pwr.edu.pl
}

of their innovation-decisions that have already been made and may reverse previous decisions if exposed to conflicting messages about the innovation (e.g. negative WOM).

The contribution of this paper is twofold. Firstly, in contrast to classical models of innovation diffusion [9], we propose a model that is able to describe not only a successful diffusion but also a situation in which the innovation is not able to conquer the market. The key question that we ask here is: What mechanism on the microscopic level can hamper innovation diffusion? Secondly, by introducing the concept of the reservation price, we make a first step towards investigating the demand curve for this product, which is one of the fundamental issues in the economy and is studied in [10].

\section{The model}

We consider a system of $N$ agents, i.e. potential customers of an innovation. In the text, we use the words "agent", "individual", "customer" and "consumer" interchangeably with spinson, a term coined by Nyczka and Sznajd-Weron [11] that nicely allows to go around gender issues and reflects the dyadic nature of an agent's opinion $(\rightarrow$ spin) and the object of study ( $\rightarrow$ person). The agents are connected by undirected links and form a connected graph. Although the model is general, in this study we restrict ourselves to fully connected networks, which can represent cliques in the society and where each agent is connected to every other agent in the system (also known as complete graphs). Such an approach allows for analytical treatment, which is particularly useful at an initial stage, when we want to identify the role of each of the model parameters. Extended studies, also for real Facebook and Google + social networks are presented in [10].

Each spinson $(i=1, \ldots, N)$ is described by two traits: $P_{i} \in[0,1]-$ a continuous variable that represents the reservation price, and $S_{i}(P) \in\{0,1\}$ - a binary opinion, that depends on the product's market price, $P$. Opinion $S_{i}$ of the $i$-th spinson takes value 1 if the market price is lower or equal to its reservation price: $P \leq P_{i}$, 
i.e. if the spinson is willing to adopt and buy the product, and 0 otherwise. As is typical in the innovation diffusion literature, the main object of study here is the penetration rate or penetration level, i.e. the percentage of adopted spinsons as a function of model parameters: $Q \equiv Q(\cdot)$.

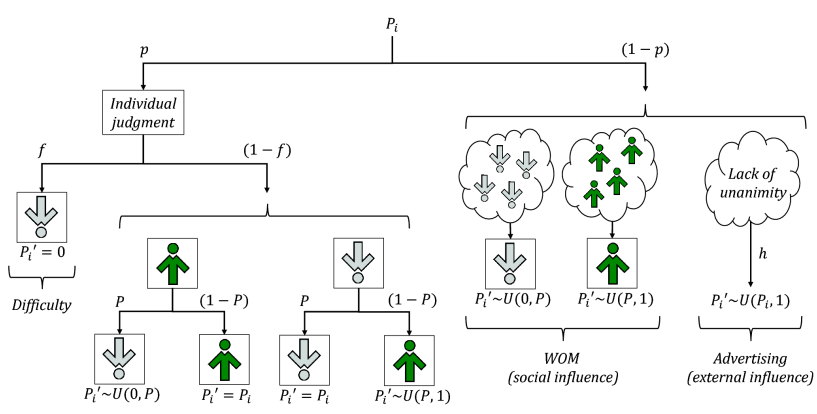

Fig. 1. A graphical representation of the evolution of an agent's reservation price within an elementary time step $\Delta t$. The icon and the term used to refer to an agent - spinson - reflect the dyadic nature of an agent's opinion $(\rightarrow \operatorname{spin})$ and the object of study ( $\rightarrow$ person). $P_{i}$ denotes the reservation price of spinson $i$ at time $t$, whereas $P_{i}^{\prime}$ the reservation price at time $t+\Delta t$. Arrow labels denote occurrence probabilities; arrows without labels indicate a probability of one. Notation $X \sim$ $U(a, b)$ means that variable $X$ takes a random value from the uniform distribution on the interval $(a, b)$. See text for details.

The evolution of reservation prices is governed by two different processes: (i) individual (or personal) judgment and (ii) social network and mass-media influence. By individual (or personal) judgment we understand the evaluation of the product (i.e. the innovation) according to its non-financial costs and the benefits it brings. We assume that personal judgment is neither affected by mass-media nor by WOM, hence it is closely related to the concept of independent behavior $[12,13]$. We introduce independence in a similar way as was done in [11], i.e. each spinson behaves independently with probability $p$ and forms an opinion solely on the basis of individual judgment, see the left part of Fig. 1. On the other hand, with probability $(1-p)$ the spinson is exposed to social influence (i.e. WOM) and advertising, see the right part of Fig. 1. One could argue that assigning the same value of $p$ to each agent is not realistic and should rather be taken from some distribution. However, to the best of our knowledge, the form of this distribution is not known [14]. In the absence of this knowledge, Sznajd-Weron et al. [15] have considered two extreme approaches - situation (homogeneous agents) and person (a binomial distribution of independence) - and shown that the former is more realistic. Therefore we use it also in this study. However, we are aware that probably something in between would be more appropriate. The question how such a distribution influences results of our model is left for future research. For now, let us assume that $p$ is an average level of independence in the society and that the variance of the distribution of independence is small. Preliminary results show that in such a case the outcomes are qualitatively the same as for a constant value of $p$.

Although innovative products are assumed to be beneficial, it is possible that a spinson will reject it if the potential behavioral costs outweigh the gains. This means that some consumers may find the product redundant, useless and not needed and hence will not accept it even when it is offered for free. In such a case, the spinson will assign a reservation price $P_{i} \rightarrow P_{i}^{\prime}=0$, where $P_{i}$ denotes the reservation price of spinson $i$ at time $t$, while $P_{i}{ }^{\prime}$ the reservation price at time $t+\Delta t$. In order to capture the adoption difficulty, the probability of a negative product evaluation is described by parameter $f$. The way we perceive difficulty is similar to [16].

On the other hand, with probability $(1-f)$ the spinson will evaluate the product positively, because the benefits of adopting the innovation exceed its non-financial costs. This creates the possibility of revising the current product appraisal. We assume that: (i) a spinson assigns a new reservation price only when it is associated with a change of the current opinion, $S_{i}(P)$, and (ii) the probability of the opinion change depends on the market price, $P$. The second assumption follows from the fact that a consumer is more likely to switch to a positive opinion, when the market price is low than when it is high. Hence, two situations are possible. With probability $P$, an agent will set $S_{i}(P) \rightarrow S_{i}{ }^{\prime}(P)=0$ and become a "gray" spinson in Fig. 1. If this action is associated with an opinion change (i.e. $S_{i}(P)=1$ and the spinson was "green") then the agent randomly draws a new reservation price from $U(0, P)$, i.e. the uniform distribution on the interval $(0, P)$. On the other hand, with probability $(1-P)$, an agent will set $S_{i}(P) \rightarrow S_{i}{ }^{\prime}(P)=1$ and become a "green" spinson in Fig. 1. If this action is associated with an opinion change (i.e. $S_{i}(P)=0$ and the spinson was "gray") then the agent randomly draws a new reservation price from $U(P, 1)$.

Social influence is a result of interactions between a spinson and a group of neighboring (i.e. directly connected) spinsons in a given time step. Following $[5,11,15]$, we assume that a spinson interacts with a set of $q$ neighbors (i.e. a $q$-lobby) instead of all of its neighbors. It has been observed in a number of social experiments that for social influence it is not only essential for the majority to be of sufficient size but also unanimous [17]. Hence, if at least one of $q$ selected nearest neighbors shares the opinion of the spinson, WOM will have no effect on the reservation price. However, when a spinson is confronted by a unanimous group of $q$ neighboring spinsons sharing the opposite opinion, it will conform to peer pressure [13] and choose a new reservation price. It should be emphasized that social influence can be both positive and negative, depending on the opinion distribution in the $q$-lobby. When all $q$ selected neighbors agree that the product is worth its price, the spinson will randomly draw a new reservation price from $U(P, 1)$. 
On the contrary, when all $q$ selected neighbors admit that the market price is too high, the spinson will randomly draw a new reservation price from $U(0, P)$.

In the remaining situations, when the $q$-lobby is not unanimous, the potential consumer is exposed to advertising. The impact of advertising is expressed by parameter $h$, the probability that a spinson increases the product evaluation due to new information obtained from massmedia. In such a case, the spinson will randomly draw a new reservation price from $U\left(P_{i}, 1\right)$. Note that the mass-media influence is heterogeneous and depends on the current reservation price of the spinson.

\section{Extensions of the previous model}

The model we present here is an extended version of the model of innovation diffusion proposed by Przybyła et al. [4] and applied to modeling of the adoption of dynamic electricity tariffs by Kowalska-Pyzalska et al. [5]. There are two major modifications. Firstly, we make use of the concept of reservation prices, which is very important from the economic point of view [6]. Unfortunately, this modification introduces a new parameter - market price $P$ - to the model. Because the dependence on the remaining parameters has been extensively studied in [4], here we concentrate on the role of $P$. Secondly, we consider the size of the influence group, $q$, as a parameter. Such a generalization has been studied in the absence of the external field [11], but in case of innovation diffusion only a single value of $q=4$ has been considered $[4,5]$. Here, we briefly discuss also the role of $q$, because we believe that next to the optimal product price this knowledge could also be useful in building marketing strategies.

From the perspective of social influence the optimal group size has been identified to vary between three and four agents, depending on the experiment [17]. This could suggest that the previously used value of $q=4$ is the most suitable to model social systems. However, it is not clear if the optimal group size, from the perspective of the social influence, is also the most common size of naturally occurring groups. Many different studies, conducted for over the last 60 years, have shown that across different informal locations, like side walks, stores, playgrounds, carnivals, receptions, swimming pools, basketball games intermissions, church socials and train depots, freely-forming groups ranged in size from two to seven [18]. Moreover, when opinions can be expressed privately (without the group knowing your opinion), the role of the group size increases with $q$ also for $q>4$ [17]. Therefore, we study the model using three different group sizes $q \in\{3,4,8\}$.

\section{Mean-field analytics}

To study the time evolution of the market penetration rate we can use the method known in statistical physics as the mean-field approach (MFA). On a complete graph each agent is connected with every other agent and therefore they are all neighbors. Hence, the system is completely homogeneous in the sense that the local concentration of adopted agents is statistically equal to the global, system-wide concentration.

Recall, that in our model in a single, elementary time step $\Delta t$ the penetration rate can increase by $\frac{1}{N}$ with $\operatorname{Prob}\left\{Q \rightarrow Q^{\prime}=Q+\frac{1}{N}\right\} \equiv \gamma^{+}$and decrease by $\frac{1}{N}$ with $\operatorname{Prob}\left\{Q \rightarrow Q^{\prime}=Q-\frac{1}{N}\right\} \equiv \gamma^{-}$. Now, following the argumentation in $[4,15]$, these probabilities can be written in the following form:

$$
\begin{aligned}
\gamma^{+} & =\underbrace{p(1-f)(1-Q)(1-P)}_{\text {individual judgment }}+\underbrace{(1-p) Q^{q}(1-Q)}_{\text {WOM }} \\
& +\underbrace{(1-p)\left\{1-Q^{q}-(1-Q)^{q}\right\} h\{(1-Q) \phi\}}_{\text {advertising }}, \\
\gamma^{-} & =\underbrace{p(1-f) Q P+p f Q}_{\text {individual judgment }}+\underbrace{(1-p)(1-Q)^{q} Q}_{\text {WOM }},
\end{aligned}
$$

see also Fig. 1. Moreover, $\phi$ is the probability that an agent will evaluate the product positively after massmedia influence. This probability can be approximated by

$$
\phi \approx \frac{1}{P} \int_{0}^{P} \frac{1-P}{1-P_{i}} \mathrm{~d} P_{i}=\frac{P-1}{P} \log (1-P),
$$

which is exact under the assumption of uniformly distributed reservation prices $P_{i}$.

\section{Results}

We focus on measuring two basic quantities in the field of innovation diffusion $[1,9]$, namely:

- Market penetration rate $Q$ - the percentage of adopted spinsons until time $t$. In case of a successful innovation, $Q(t)$ is believed to take the form of an S-shaped curve.

- First-time adopters $\Delta Q$ - the percentage of spinsons that is adopted at time $t$, which in a discrete time setup is simply the difference in the market penetration rate at two consecutive time steps: $\Delta Q(t)=Q(t)-Q(t-1)$.

The latter quantity is interesting because it can reveal several scenarios, which are hidden behind the cumulative S-shaped adoption curve, such as (i) a rapid initial growth followed by a much slower decay $[3,19]$, (ii) a symmetrical growth and decay, characteristic for the famous Bass model [20], (iii) a slow initial growth followed by a much faster decay (not observed up until now) or (iv) a characteristic saddle between two peaks [3]. We will see that within our model we can obtain a whole plethora of possibilities.

In Fig. 2 we present $Q(t)$ and $\Delta Q(t)$ for three group sizes $q=3,4,8$, a fixed set of parameters $(p=0.05$, $f=0.5, h=0.2$ ) and two market prices: $P=0.26$ and $P=0.18$. The time evolution of $Q(t)$ for a whole range of market prices is shown in Fig. 3. However, already in Fig. 2 we can observe several interesting results. 

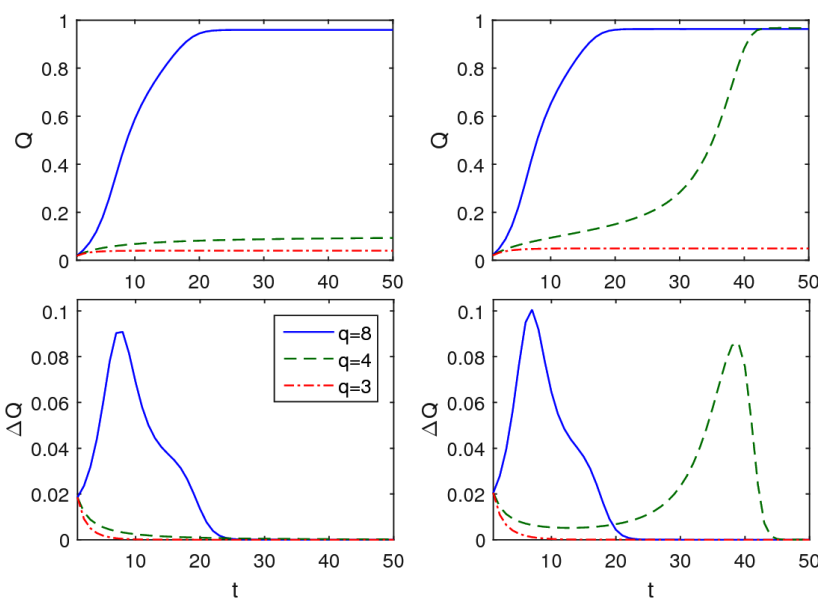

Fig. 2. Market penetration rate $Q$ as a function of time $t$ for three group sizes $q=3,4,8, p=0.05, f=0.5$, $h=0.2$ and two market prices: $P=0.26$ (left parts) and $P=0.18$ (right parts). The corresponding curves of first-time adopters $\Delta Q$ are plotted in the lower parts.
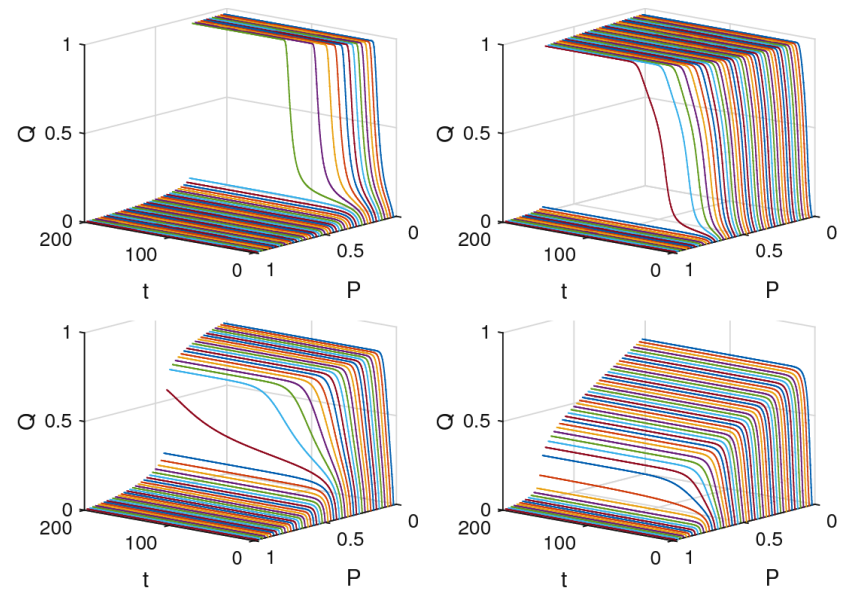

Fig. 3. Market penetration rate $Q$ as a function of time $t$ and market price $P$ for $f=0.5, h=0.2$. Results presented in the upper parts are characterized by the same value of independence $(p=0.05)$ but differ in the size of the group: $q=4$ (left part) and $q=8$ (right part). Similarly, results presented in the bottom parts are characterized by the same value of independence $(p=0.2)$ but differ in the size of the group: $q=4$ (left part) and $q=8$ (right part). Colors indicate different values of $P=0.02,0.04, \ldots, 0.98$.

Firstly, that with the increase of group size $q$, the diffusion of innovation is easier, i.e. takes place already for a higher market price $P$. Secondly, that indeed several scenarios for the first-time adopters curve are possible, as observed empirically in $[3,19]$. Results presented here suggest also that there is a critical price, above which the innovation will not spread in the market. To see this more clearly we present in Fig. 3 the market penetration rate for a whole range of market prices.

Again, we only present results for a selected set of model parameters: $h=0.2, f=0.5, p=0.05,0.2$.
However, extended studies on the role of these parameters have been presented in our previous paper [4]. Here, we focus mainly on presenting the role of the market price. As we can see, there is indeed a critical value above which the innovation does not diffuse. As usual, a critical slowing down is seen, which means that at the critical point the diffusion is very slow. Moreover, we see that not only a larger size of the group $q$, but also a higher value of independence $p$, facilitates diffusion. Of course, also a higher value of external field $h$ helps the innovation to diffuse, but this is clear and has been already discussed in $[4,5]$.

In Introduction we have asked the question What mechanism on the microscopic level can hamper innovation diffusion? The market price, $P$, is a macroscopic variable, which can be set by the producer (at least in a noncompetitive market). Similarly the external field, $h$, related usually with advertisement $[1,9]$ is a macroscopic variable, to some extent controllable by the company introducing the innovation. Also the difficulty, $f$, has been postulated as an objective, person-independent quantity [16], although it might be questionable and not very intuitive. However, definitely the independence parameter, $p$, and the size of the influence group, $q$, are microscopic variables. Both are described by a certain distribution related to the situation, culture, place, etc. [14, 18] and cannot be influenced by the producer so easily. Here, we have used one value of $p$ and one value of $q$ for the whole society, which has to be understood as an average value. We have shown that the critical price, below which diffusion will spread increases with both parameters diffusion is easier for larger groups and higher values of independence. However, the role of independence is not unambiguous - a higher value of $p$ supports diffusion in a sense that the take-off is possible for a higher market price $P$, but on the other hand for higher values of $p$ the innovation does not conquer the market completely (see the bottom parts in Fig. 3).
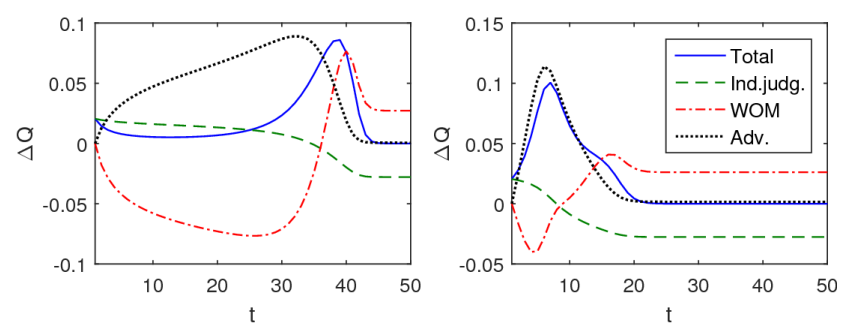

Fig. 4. Curves of first-time adopters $\Delta Q$ ("Total") and its three components: "Individual judgment", "WOM" and "Advertising", see formulae (1)-(2), as a function of time $t$ for two group sizes $q=4$ (left part) and 8 (right part) and $p=0.05, f=0.5, h=0.2, P=0.18$ (as in the right parts of Fig. 2).

Such results can be also understood heuristically. Independence introduces noise to the system, i.e. destroys order. In the initial state all spinsons are unadopted and therefore independence is needed to activate changes in 
the system. Without independence the system would stay forever in the initial unadopted state. On the other hand, the system will never reach a fully adopted state, because independence destroys symmetrically both orders - fully unadopted and fully adopted states. The role of the size of the group of influence is less intuitive. However, if we recall that in our model a unanimous $q$-panel is needed for social influence (WOM), then it becomes clear that if $q$ is large, social influence is less probable and spinsons are influenced more by advertising, which flips them into the adopted state. This is visible in Fig. 4 in which first-time adopters are divided into three groups - those driven by individual judgment, WOM, and advertising. For $q=4$ innovation diffusion takes off when WOM starts to increase, which means that in this case the diffusion is driven mainly by social influence. However, for $q=8$ the situation is completely different. The maximum speed of diffusion, $\max (\Delta Q)$, coincides with minimum WOM, which shows that in this case social influence is not the force that drives innovation diffusion. Instead, the dotted black curve represents the rate of first-time adopters driven by advertising coincides with the solid blue curve of the total rate of first-time adopters.

\section{Conclusions}

When analyzing the effect of WOM on the market behavior of a producer, we should not restrict ourselves only to the positive impact. Numerous studies have indicated that the positive and negative WOM coexist and influence the opinion formation. Moreover, it is well known, although not deeply studied, that the adoption process may be discontinued due to various reasons, such as replacement or disenchantment [1]. In our model, the innovation adoption is governed by three processes: individual judgment, social influence (WOM), and advertising. However, only the mass-media effect is always positive and supports the diffusion. When WOM is considered, agents are allowed to spread both positive and negative opinions. Hence, the dissatisfied individuals can discourage others from adopting the innovation, like in [7]. We have demonstrated that when the discontinuation of adoption is allowed then two different faces of WOM, positive and negative, are revealed. This outcome expands the findings of [2], who discussed only the negative impact of WOM.

Moreover, we have introduced the concept of the reservation price, which is well known in the economic literature, but has not been explored in the context of opinion dynamics models. This is a significant step forward since the infection-spreading or percolation-type models that have been used so far are not consistent with the experimental data on innovation diffusion [21].

Finally, we have found out that with the increase of the size of the group of influence the diffusion is easier, smoother, and faster. It takes place even for higher market prices. We have also observed that the market price of the good really matters: there exists a critical price above which the innovation will not spread and below which it will conquer the market. These findings are particularly useful for producers who can take them into account when designing marketing strategies for their innovative products.

\section{Acknowledgments}

We are grateful to an anonymous reviewer for inspiring comments. This work was supported by the National Science Center (NCN, Poland) through grant no. 2013/11/B/HS4/01061.

\section{References}

[1] E.M. Rogers, Diffusion of Innovations, The Free Press, New York, NY 2003.

[2] J. Goldenberg, B. Libai, E. Muller, Int. J. Res. Market. 27, 4 (2010).

[3] R. Peres, E. Muller, V. Mahajan, Int. J. Res. Market. 27, 91 (2010).

[4] P. Przybyła, K. Sznajd-Weron, R. Weron, Adv. Complex Syst. 17, 1450004 (2014).

[5] A. Kowalska-Pyzalska, K. Maciejowska, K. Suszczyński, K. Sznajd-Weron, R. Weron, Energy Policy 71, 164 (2014).

[6] H.R. Varian, Intermediate Microeconomics. A Modern Approach, 8th ed., W.W. Norton and Co., New York, NY 2010.

[7] G. Deffuant, S. Huet, F. Amblar, Am. J. Sociol. 110, 1041 (2005).

[8] J. Goldenberg, B. Libai, E. Muller, Market. Lett. 12, 211 (2001).

[9] E. Kiesling, M. Güntherand, C. Stummer, L.M. Wakolbinger, Centr. Europ. J. Operat. Res. 20, 183 (2012).

[10] K. Maciejowska, A. Jędrzejewski, A. KowalskaPyzalska, R. Weron, Acta Phys. Pol. A 129, 1045 (2016).

[11] P. Nyczka, K. Sznajd-Weron, J. Statist. Phys. 151, 174 (2013).

[12] P.R. Nail, S.I. Di Domenico, G. MacDonald, Rev. Gen. Psychol. 17, 1 (2013).

[13] P.R. Nail, K. Sznajd-Weron, Acta Phys. Pol. A 129, 1050 (2016).

[14] K. Byrka, A. Jędrzejewski, K. Sznajd-Weron, R. Weron, HSC Research Report 15/10, 2015.

[15] K. Sznajd-Weron, J. Szwabiński, R. Weron, PLoS ONE 9, 1 (2014).

[16] F.G. Kaiser, K. Byrka, T. Hartig, Personal. Soc. Psychol. Rev. 14, 351 (2010).

[17] R. Bond, Group Process. Intergroup Relat. 8, 331 (2005).

[18] R. Barry Ruback, R.T. Collins, S. Koon-Magnin, Weina Ge, L. Bonkiewicz, C.E. Lutz, Environm. Behav. 45, 239 (2013).

[19] M. Günther, C. Stummer, L.M. Wakolbinger, M. Wildpaner, J. Operat. Res. Soc. 62, 12 (2011).

[20] F.M. Bass, Manag. Sci. 15, 215 (1969).

[21] C.H. Weiss, J. Poncela-Casasnovas, J.I. Glaser, A.R. Pah, S.D. Persell, D.W. Baker, R.G. Wunderink, L.A. Nunes Amaral, Phys. Rev. X 4, 041008 (2014). 